
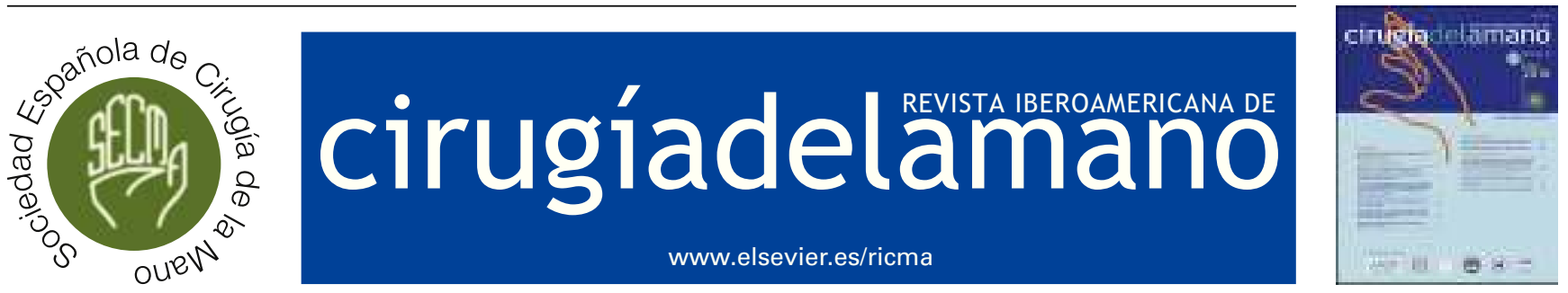

ARTÍCULO ORIGINAL

\title{
Artrodesis radio-escafo-lunar. Experiencia en el ámbito laboral
}

\author{
C. Martínez Nisa*, E. Gárate Otaola, T. Blázquez Martín, P. Carretero Cristóbal, \\ M. Dodangeh González y J. Castaño Lloris
}

Unidad de Cirugía de Mano y Microcirugía, Hospital Monográfico ASEPEYO de Cirugía Ortopédica, Traumatológica y Rehabilitación, Coslada, Madrid, España

Recibido el 2 de febrero de 2016; aceptado el 9 de marzo de 2016

Disponible en Internet el 6 de abril de 2016

\section{PALABRAS CLAVE \\ Artrodesis; \\ Radio-escafo-lunar; Agujas de Kirschner; Artrosis radiocarpiana}

\begin{abstract}
Resumen
Introducción: La artrodesis radio-escafo-lunar (RSL) es un procedimiento quirúrgico paliativo utilizado para el tratamiento de artrosis radiocarpiana en pacientes con la articulación mediocarpiana preservada. Esta técnica reduce el dolor asociado a la artrosis radiocarpiana y preserva la función de la articulación mediocarpiana.

Material y método: Se realizó un estudio descriptivo retrospectivo, de una serie de 45 pacientes intervenidos en nuestro hospital desde 2005 a 2015. En todos los casos se llevó a cabo la intervención quirúrgica mediante artrodesis RSL con agujas de Kirschner (AK) e injerto autólogo de cresta ilíaca. Se evaluó la fuerza, rango de movilidad, dolor, tasas de seudoartrosis y de conversión a artrodesis total y el tipo de incapacidad que obtuvo el paciente al final del proceso.

Resultados: Se obtuvo un déficit de fuerza de puño del $49 \%$ respecto a la mano contralateral. El rango de movilidad postoperatorio fue de $20^{\circ}$ flexión, $25^{\circ}$ extensión, $7^{\circ}$ desviación radial y $14^{\circ}$ desviación ulnar. Se obtuvo un resultado favorable en cuanto a la reducción del dolor, según la escala analógica visual (EVA) posquirúrgica. La tasa de conversión a artrodesis total fue de un $15,5 \%$ y el fracaso de consolidación de un $13,3 \%$.

El $83 \%$ de los casos obtuvo una incapacidad parcial y el $27 \%$ una incapacidad total. Conclusión: La artrodesis RSL en una técnica quirúrgica paliativa satisfactoria para el tratamiento del dolor que obtiene una tasa de consolidación alta y que mantiene la movilidad de la articulación mediocarpiana. Sin embargo, la mayoría de los pacientes obtienen algún tipo de incapacidad laboral tras su realización.

() 2016 SECMA. Publicado por Elsevier España, S.L.U. Este es un artículo Open Access bajo la licencia CC BY-NC-ND (http: //creativecommons.org/licenses/by-nc-nd/4.0/).
\end{abstract}

\footnotetext{
* Autor para correspondencia.

Correo electrónico: cristinamnisa@gmail.com (C. Martínez Nisa).
} 


\section{KEYWORDS}

Arthrodesis;

Radioscapholunate;

Kirschner wires;

Radiocarpal arthritis
Radioscapholunate arthrodesis. Experience in the workplace

\begin{abstract}
Introduction: Radioscapholunate (RSL) arthrodesis is an effective procedure for the treatment of isolated and non-salvageable radiocarpal arthritis. This procedure reduces pain associated with radiocarpal arthritis, preserving mid-carpal joint function

Material and method: A descriptive retrospective study was conducted on 45 patients treated with radioscapholunate arthrodesis in our hospital from 2005 to 2015. Kirschner wires were used in all cases to temporarily fix the arthrodesis and autologous bone graft from the iliac crest.

Results: A decrease of $49 \%$ decrease in grip strength was observed compared with the contralateral hand. The range of motion was $20^{\circ}$ of flexion, $25^{\circ}$ of extension, $7^{\circ}$ of radial deviation, and $14^{\circ}$ of ulnar deviation. Favourable outcomes were obtained according to the post-operative Visual Analogue Scale (VAS). The percentage of conversion to total wrist arthrodesis was $15.5 \%$, and the non-union rate was $13.3 \%$.

Conclusions: Radioscapholunate arthrodesis is an effective procedure for improving pain with a high consolidation rate that preserves mid-carpal movement. However, most of the patients obtained an incapacity for work after the procedure.

(c) 2016 SECMA. Published by Elsevier España, S.L.U. This is an open access article under the CC BY-NC-ND license (http://creativecommons.org/licenses/by-nc-nd/4.0/).
\end{abstract}

\section{Introducción}

La artrosis radiocarpiana es el resultado de la degeneración articular como consecuencia de etiología inflamatoria, metabólica o postraumática, siendo esta última la más frecuente dentro del medio laboral. Se considera la causa más común de dolor e incapacidad funcional a nivel de la muñeca.

La artrodesis radio-escafo-lunar (RSL) es una técnica quirúrgica paliativa para aquellas muñecas con artrosis radiocarpiana sin afectación de la articulación mediocarpiana. Esta técnica quirúrgica fue inicialmente descrita por Gordon y King en $1961^{1}$ con el objetivo de disminuir el dolor radiocarpiano, estabilizando las articulaciones lesionadas, dolorosas o inestables a costa de limitar el rango de movilidad articular que se mantiene gracias a la articulación mediocarpiana.

Las indicaciones de esta técnica quirúrgica ${ }^{2}$ son las muñecas con artrosis radiocarpiana dolorosa, siempre y cuando la articulación mediocarpiana se encuentre preservada. Entre ellas destacan por frecuencia las secuelas de fracturas intraarticulares del radio distal. Según un artículo publicado por Jupiter y $\mathrm{Knirk}^{3}$, el 91\% de este tipo de fracturas con incongruencia articular evolucionan a cambios degenerativos a dicho nivel y por lo tanto en posterior artrosis radiocarpiana (fig. 1). Otra etiología también frecuente son las muñecas con lesiones tipo «scaphoid nonunion advance collapse» (SNAC), es decir, con un colapso avanzado del carpo por pseudoartrosis de escafoides, o las tipo «scapholunate advance collapse» $(\mathrm{SLAC})^{4}$, debidas a lesiones de la escafolunar con inestabilidad en flexión y pronación del escafoides, las cuales cursan con dolor mecánico y pérdida de movilidad de muñeca en todos los planos. Las lesiones tipo SLAC fueron descritas por Watson en $1984^{5}$ como la causa más frecuente de artrosis degenerativa de la muñeca (55\%). En los casos de muñecas con lesión tipo SNAC o SLAC, se consideran indicación de artrodesis RSL aquellas en estadíos precoces, puesto que en los más avanzados la articulación mediocarpiana ya está afectada. Así mismo, esta técnica quirúrgica paliativa también puede estar indicada en los estadios iniciales de necrosis avascular del semilunar o enfermedad de Kiënbock. También existe indicación en aquellas muñecas con artrosis debida a enfermedades por depósito o reumáticas con la articulación mediocarpiana preservada.

La movilidad postoperatoria publicada ${ }^{6}$ varía dependiendo de si se realiza o no algún gesto quirúrgico asociado, como la resección del polo distal del escafoides (DSE), como posteriormente comentaremos. Los resultados publicados en la literatura sobre la tasa de consolidación varían según la síntesis empleada, bien sea mediante agujas de Kirschner $(\mathrm{AK})$, placa en «T», tornillos de compresión o grapas ${ }^{7}$.

El objetivo de este trabajo consiste en presentar los resultados clínicos y radiológicos de los pacientes intervenidos mediante artrodesis RSL realizadas en nuestro centro en aquellas muñecas con artrosis radiocarpiana y articulación mediocarpiana conservada, durante un período de 10 años.

\section{Material y método}

Realizamos un estudio descriptivo retrospectivo en 45 pacientes intervenidos en nuestro hospital desde enero de 2005 hasta enero de 2015 por el mismo equipo. Los criterios de inclusión de este estudio fueron pacientes en edad laboral, con patología degenerativa radiocarpiana y sin afectación de la articulación mediocarpiana en el momento del diagnóstico, a los cuales se les intervino de artrodesis RSL. Los criterios de exclusión fueron, por consiguiente, degeneración de la articulación mediocarpiana en el momento del diagnóstico.

La edad media de los pacientes fue de 40 años (entre 27 y 58 años). En cuanto a la lateralidad, lo más frecuente fue el lado dominante en un $64 \%$ respecto al no dominante en un $36 \%$. En relación con el género de los pacientes, predominó el masculino (84\%) sobre el femenino (16\%). Entre 

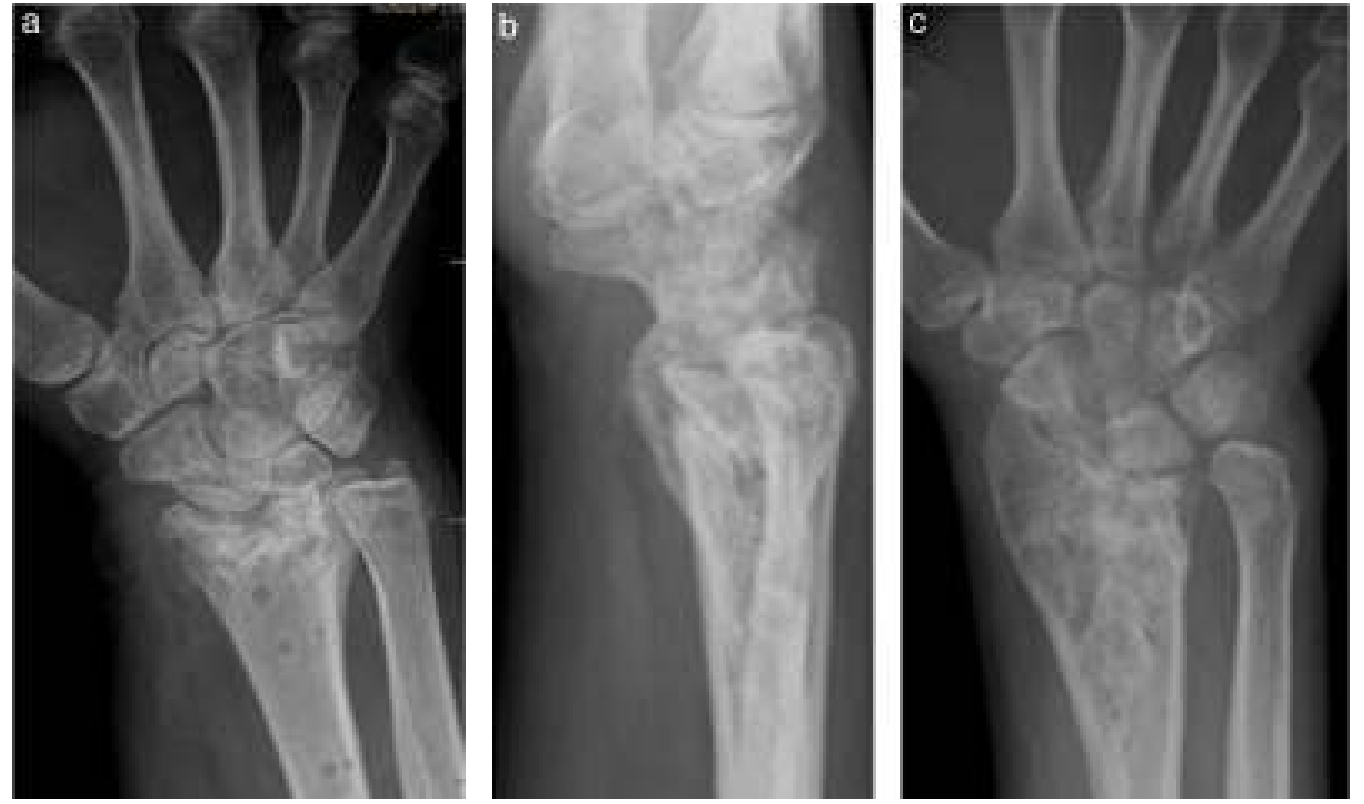

Figura 1 a) Imagen radiográfica anteroposterior de paciente con antecedentes de fractura intraarticular de radio distal tratada con fijador externo que evolucionó a lesión tipo SLAC. b y c) Imagen radiográfica lateral y anteroposterior de paciente con lesión tipo SNAC tras fractura intraarticular de radio tratada mediante osteosíntesis con placa de radiodistal.

las profesiones desempeñadas previas a la cirugía destacaron los trabajadores manuales y de fuerza tales como mecánico, agricultor, chófer y albañil. El tiempo medio de hospitalización fue de 3 días ( 2 a 4 días). La rehabilitación postoperatoria fue de 9 meses de media, con un período comprendido entre 4 y 12 meses. El tiempo medio de seguimiento fue de 32 meses (entre 18 y 96 meses).

Las variables medidas fueron: a) fuerza postoperatoria y al finalizar la rehabilitación, medida con un dinamómetro electrónico (aplicación Ned Discapacidad/IBV con instrumento modulador específico de mano Ned-VEP/IBV) para la fuerza obtenida mediante los test isocinéticos e isométricos (fuerza máxima isométrica, trabajo isocinético y déficit global de fuerza, así como la función de muñeca y mano) comparada con la mano contralateral; b) el rango de movilidad postoperatorio y el dolor, medido mediante la escala analógica visual (EVA); c) el porcentaje de reartrodesis por seudoartrosis y de conversión a artrodesis total; d) el tiempo de baja, y e) el porcentaje de pacientes que recibieron incapacidad total o parcial postoperatoria.

El resultado laboral se realizó valorando la incorporación al trabajo del paciente siguiendo la escala del Instituto Nacional de la Salud (INS) que determina diferentes categorías según considere al paciente como: curado, con secuelas baremables, incapacidad permanente parcial (IPP) si ocasiona al trabajador una disminución no inferior al $33 \%$ en el rendimiento para su profesión, incapacidad permanente total (IPT) si inhabilita al trabajador para su profesión habitual pero puede dedicarse a otra distinta, incapacidad absoluta (IA) si inhabilita al trabajador para toda profesión u oficio, o gran invalidez (GI) si el trabajador incapacitado permanente necesita la asistencia de otra persona para los actos más esenciales de la vida $^{8}$.

\section{Técnica quirúrgica}

Bajo anestesia general con el paciente en decúbito supino e isquemia preventiva en el miembro superior indicado, se realizó abordaje dorsal en «S» itálica a nivel radiocarpiano. A continuación se seccionó el retináculo extensor a lo largo del tercer y cuarto compartimentos; en el suelo de este último se realizó neurectomía del nervio interóseo posterior y se realizó la neurectomía del mismo.

Mediante capsulotomía dorsal transversa con «flaps» longitudinales se accedió a la articulación radiocarpiana (modificación del abordaje de Berger y Bishop) ${ }^{9}$; de esta forma se refrescaron las superficies articulares del radio, escafoides y semilunar hasta observar hueso esponjoso.

A continuación se extrajo injerto óseo autólogo esponjoso de cresta ilíaca que se aportó a la articulación radiocarpiana para su artrodesis.

Se realizó fijación de escafoides y semilunar en $20^{\circ}$ de extensión con $2 \mathrm{AK}$ de 1,5 mm cada una (fig. 2). La primera se introdujo desde el dorso del radio al escafoides y la segunda de la zona más radial del radio al semilunar. En los casos en los que durante la cirugía se observó algún grado de inestabilidad escafolunar, se estabilizó dicha articulación con una tercera AK del mismo diámetro colocando el semilunar en posición neutra.

La DSE solo se realizó en un único caso, en una muñeca con lesión tipo SLAC. No se asoció ningún gesto quirúrgico sobre el ganchoso ni sobre el piramidal en ningún paciente.

A continuación se suturó la cápsula dorsal y el retináculo extensor, y por último se realizó el cierre de la piel. En ningún caso se dejó drenaje aspirativo en la muñeca, pero sí en todos los pacientes en la zona donante de cresta ilíaca. Todos los pacientes fueron inmovilizados con una férula antebraquiopalmar. 

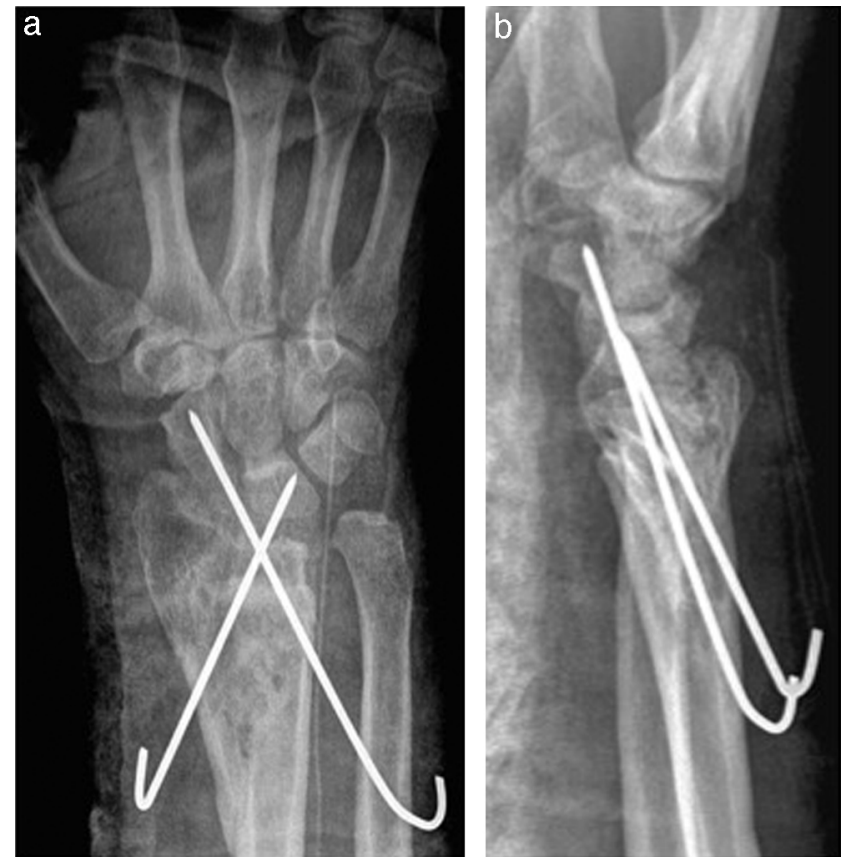

Figura 2 a y b) Radiografía anteroposterior y lateral postoperatoria de artrodesis RSL sintetizada con 2 agujas de Kirschner: radiosemilunar y radioescafoides.

\section{Manejo postoperatorio}

Se realizó un seguimiento con control evolutivo en consulta cada 3 semanas para comprobar el estado de la consolidación de la artrodesis radiocarpiana mediante radiografías en proyección anteroposterior y lateral. Una vez cumplidas las 8 semanas postoperatorias se retiraron tanto el material de osteosíntesis como la inmovilización, salvo en aquellos casos en los que las imágenes radiográficas de control mostraron cierto retraso en la consolidación de la artrodesis, en los que se optó por retirar las AK y cambiar la inmovilización a yeso antebraquial de 2 a 4 semanas más.

La rehabilitación comenzó de media a los 2,5 meses postoperatorios; primero con ejercicios pasivos asistidos $y$ posteriormente activos centrados en la movilización de la articulación mediocarpiana y en la potenciación de la fuerza prensora.

\section{Resultados}

La etiología de la artrosis radiocarpiana que precisó la artrodesis RSL fue variada. El mayor porcentaje (41\%) pertenecía a pacientes con secuela de fractura de extremidad distal del radio. El segundo grupo en frecuencia fueron aquellos con secuelas de disociación escafolunar (29\%). También se encontró una incidencia destacable en pacientes con secuelas de fractura de escafoides (14\%). El cuarto grupo lo representaron los pacientes con lesiones combinadas de factura de radio distal y disociación escafolunar (8\%). Por último, en orden de frecuencia, se encontraron pacientes con secuelas de enfermedad de Kiënbock (6\%) y lesiones combinadas de fractura de escafoides y fractura intraarticular de radio distal (2\%).
En cuanto a la fuerza resultante postoperatoria y al finalizar la rehabilitación, se observó un déficit del $49 \%$ en la mano intervenida respecto a la contralateral. El rango de movilidad postoperatorio obtenido a expensas de la articulación mediocarpiana fue de $20^{\circ}$ flexión, $25^{\circ}$ extensión, $7^{\circ}$ desviación radial y $14^{\circ}$ desviación ulnar. Aunque cierto dolor residual persistió después de la artrodesis RSL, la mayoría de los pacientes se mostraron satisfechos con los resultados en comparación con su estado preoperatorio.

La valoración del EVA postoperatorio fue mayor en aquellos pacientes en los que se observó fallo en la consolidación de la artrodesis (3,7 sobre 10$)$. Sin embargo, una vez realizado el tratamiento de dicha complicación se obtuvo un EVA final satisfactorio de 1,4.

\section{Complicaciones}

La complicación importante de mayor frecuencia fue la aparición de artrosis mediocarpiana en 7 casos (15,5\%), que precisó la conversión a artrodesis total en todos ellos.

El fracaso de la consolidación de la artrodesis ocurrió en 6 pacientes $(13,3 \%)$ y precisó la consiguiente reartrodesis por pseudoartrosis (fig. 3).

Tres de los pacientes desarrollaron síndrome de distrofia simpático refleja $(6,6 \%)$.

Hubo 2 pacientes con infección superficial de las AK, cuya resolución precisó solo curas tópicas $(4,4 \%)$, una lesión del nervio femorocutáneo en un paciente $(2,2 \%)$ y un único caso con lesión de la rama sensitiva del nervio radial $(2,2 \%)$.

Los días de baja totales fueron 268 días de media, con un número mínimo de 163 días y un máximo de 727 días. Sin embargo, el tiempo medio de baja desde la cirugía fue de 16 semanas (mínimo de 12 y máximo de 51 ).

La valoración de incapacidad con secuelas llevada a cabo por el INS fue de $83 \%$ de los casos IPP y $27 \%$ con IPT. No se concedieron incapacidades absolutas ni grandes incapacidades.

\section{Discusión}

En nuestro estudio se observó que la artrodesis RSL mediante síntesis con AK ofrece unos resultados satisfactorios en cuanto a movilidad, fuerza resultante y dolor para el tratamiento de la patología degenerativa de la articulación radiocarpiana.

Con respecto a la literatura publicada en cuanto al rango de movilidad postoperatorio, Murray ${ }^{10}$ obtiene una flexión media de $23^{\circ}, 24^{\circ}$ de extensión, $9^{\circ}$ de desviación radial y $16^{\circ}$ de desviación ulnar, así como la fuerza resultante final con respecto a la contralateral de un $51 \%$. Estos datos son medidos en aquellos pacientes en los que la síntesis de la artrodesis se realiza mediante AK, como la que realizamos en nuestro estudio, en el que encontramos unos resultados similares.

Así mismo, Mühldorfer-Fodor et al. ${ }^{11}$ obtienen una media de $28^{\circ}$ de flexión, $20^{\circ}$ de extensión, $16^{\circ}$ de desviación ulnar y $7^{\circ}$ de desviación radial, y Nalebeuff et al. ${ }^{12}$ indican que el 25-50\% de la movilidad preoperatoria es la esperable en el postoperatorio de una artrodesis RSL. 

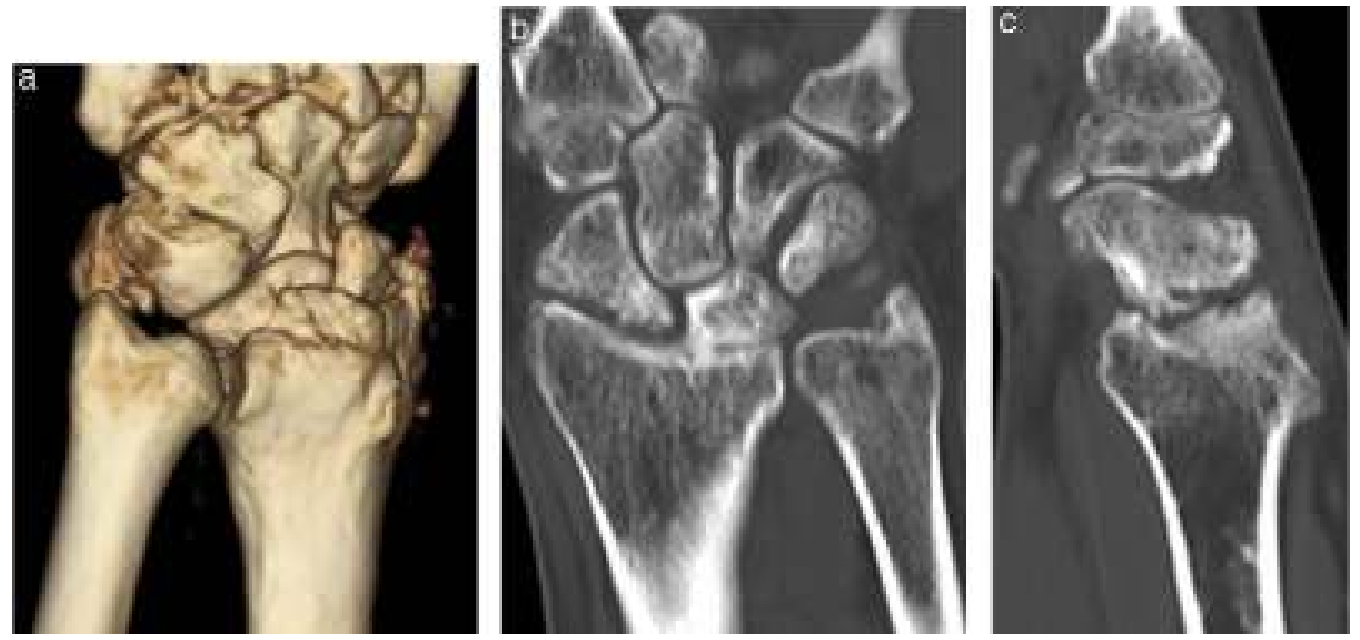

Figura 3 Imagen de tomografía computarizada (TC) en paciente intervenido de artrodesis RSL con fallo en la consolidación. a) Reconstrucción 3D donde se observa seudoartrosis dorsal radioescafoides y radiosemilunar. b) Corte coronal donde se observa fallo de consolidación en articulación radioescafoidea, semilunar y radiosemilunar. c) Corte sagital que muestra seudoartrosis radioescafoidea.

Por tanto, los resultados que obtuvimos en nuestro estudio en cuanto al rango de movilidad postoperatorio fueron similares a los publicados en la literatura al respecto.

Sin embargo, García-Elías et al. ${ }^{13}$ obtienen una mejoría en cuanto al rango de flexión y desviación radial de $14^{\circ} \mathrm{y}$ $12^{\circ}$ respectivamente, resecando el polo distal del escafoides, con una tasa de fallo en la consolidación del $0 \%$. En un período de seguimiento de 37 meses observan una incidencia menor de artrosis mediocarpiana que tras artrodesis RSL únicamente (sin DSE). Concluyen que estos mejores resultados se deben a que la articulación mediocarpiana se ve desprovista del estrés generado en la articulación escafotrapecio-trapezoidea, y por lo tanto podría disminuir la degeneración articular dolorosa de la articulación mediocarpiana a largo plazo.

No obstante, un artículo reciente publicado en 2013 por Holleran et al. ${ }^{14}$ en el que realizan estudios en cadáver comparando artrodesis RSL aislada o asociada a DSE, indican que al realizar este último gesto quirúrgico aumentan las fuerzas de rozamiento entre el semilunar y el hueso grande comparado con la artrodesis aislada, por lo que podrían provocar cambios degenerativos a largo plazo en la articulación mediocarpiana.

En nuestra serie solo se llevó a cabo la DSE en un paciente con disociación escafolunar, por lo que no podemos actualmente realizar un estudio comparativo con los resultados entre las diferentes técnicas.

Una de las complicaciones más frecuentemente asociadas a la artrodesis RSL publicada en la literatura es la tasa de pseudoartrosis, cuyo porcentaje varía según el tipo de síntesis utilizada. En el artículo publicado por Biswas et al. ${ }^{7}$ obtienen un $100 \%$ de consolidación con tornillos canulados, pero otros estudios en los que se utilizan AK obtienen un $25 \%$ de fracaso en la consolidación, como el publicado por Watson et al. en $1981^{15}$ o el artículo de Bach et al. ${ }^{16}$, con una tasa de pseudoartrosis del $11 \%$ de sus pacientes. Otros tipos de síntesis con placas en «T» realizadas por Sturznegger y Buchler presentan un $13 \%$ de tasa de no unión ${ }^{17}$. La tasa de
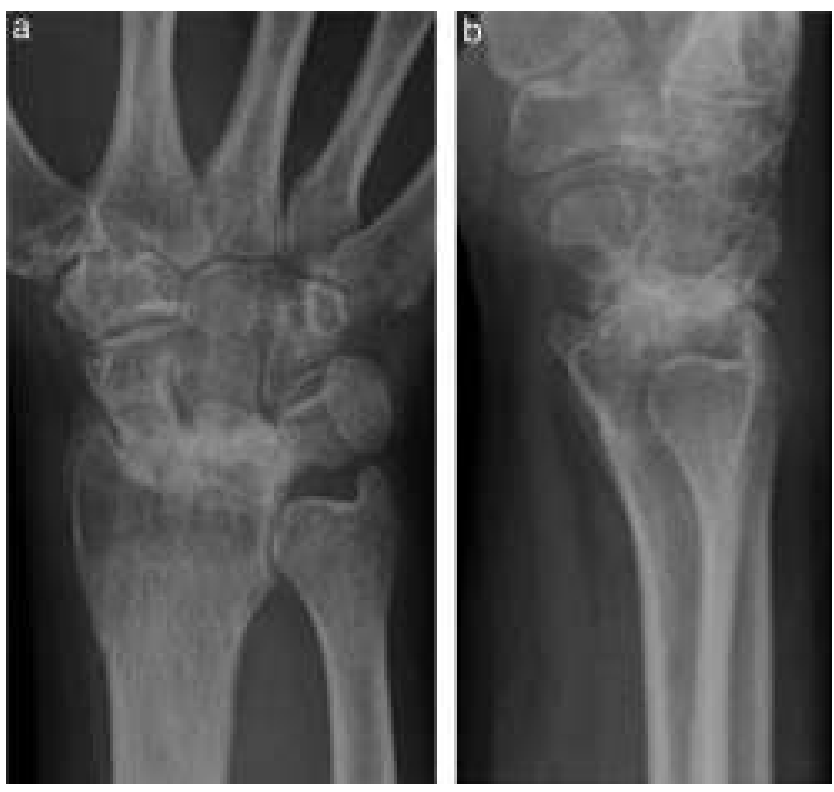

Figura 4 Imagen de radiografía anteroposterior (a) y lateral (b) en paciente intervenido de artrodesis RSL con consolidación satisfactoria.

fallo de consolidación que obtuvimos en nuestro estudio fue del $13,3 \%$, por lo tanto similar a aquellos que realizaron la síntesis con el mismo método de fijación con AK o mediante placas, grapas o tornillos de Herbert, por lo que un alto porcentaje $(86,7 \%)$ de pacientes alcanzaron una consolidación satisfactoria de la artrodesis RSL (fig. 4).

En cuanto a la conversión a artrodesis total, en nuestro estudio se llevó a cabo en 7 de 45 pacientes $(15,5 \%)$. Todos ellos presentaban artrosis en la articulación mediocarpiana y dolor incapacitante para las actividades de la vida diaria. Las etiologías que precisaron esta conversión fueron: muñecas SNAC o SLAC en 3 pacientes (43\%), lesión 
combinada de pseudoartrosis de escafoides y disociación escafolunar en 2 pacientes (29\%), fractura de extremidad distal del radio en un solo caso (14\%) y enfermedad de Kiënbock, también en un solo caso (14\%). En estos pacientes la afectación mediocarpiana era probablemente mayor de lo que parecía inicialmente en el diagnóstico, por lo que pudo no haber una adecuada indicación de artrodesis RSL.

Para la obtención de un buen resultado no solo es importante realizar una correcta técnica quirúrgica, sino también establecer la indicación adecuada en la que la articulación mediocarpiana esté preservada. Por ello es fundamental estudiar cuidadosamente dicha articulación antes de indicar una artrodesis RSL, sobre todo en aquellos pacientes con lesión tipo SNAC o SLAC grado ॥ evolucionadas en el tiempo, en las que diferenciar la afectación de la mediocarpiana mediante pruebas complementarias no es fácil. Además, al realizar una artrodesis RSL se produce una sobrecarga en la articulación mediocarpiana que puede acelerar el proceso degenerativo que pudiera existir previo a la cirugía.

El tiempo medio de baja en nuestro estudio osciló alrededor de 9 meses. En la mayoría de los pacientes, el tiempo prolongado de recuperación fue consecuencia de otras lesiones asociadas que retrasaron la cirugía o el alta laboral. Los días de baja desde la intervención de artrodesis RSL variaron alrededor de los 4 meses de media.

En cuanto a la valoración de incapacidad llevada a cabo por el INS, en nuestra serie, el $100 \%$ de los pacientes presentaron secuelas que justificaron la obtención de una IPP o IPT. Esta última indica una incapacidad para realizar su trabajo habitual y la necesidad de cambiar por un trabajo distinto con menor demanda funcional. Es importante señalar que en nuestro estudio el porcentaje de IPT fue mayor debido a que se concedió a aquellos pacientes que asociaban otras lesiones por politraumatismo. La IPP la obtuvieron los pacientes sin otra patología asociada, únicamente debida a la limitación de movilidad y pérdida de fuerza propia propia de la artrodesis RSL.

Obtuvimos unos resultados similares a la literatura publicada en cuanto al rango de movilidad postoperatorio, a la disminución del dolor valorado con la EVA, al porcentaje de fallo de consolidación en aquellos estudios en los que la síntesis se realiza mediante AK y a la tasa de conversión a artrodesis total. En estas 2 últimas variables, así como en el rango de movilidad postoperatorio, obtuvimos peores resultados que los publicados en aquellos pacientes en los que se realiza DSE.

\section{Conclusiones}

Podemos concluir, en nuestro estudio y sobre la base de nuestros resultados, que la artrodesis RSL mediante injerto autólogo esponjoso de cresta ilíaca y síntesis con AK es una técnica quirúrgica paliativa satisfactoria para el tratamiento del dolor secundario a diversas patologías de la articulación radiocarpiana siempre y cuando se mantenga preservada la mediocarpiana; por ello es prioritario realizar una correcta indicación quirúrgica.

Consideramos que es necesario realizar estudios a más largo plazo sobre los efectos en la articulación mediocarpiana tras la artrodesis RSL con y sin DSE añadida.

\section{Conflicto de intereses}

Los autores declaran no tener ningún conflicto de intereses.

\section{Agradecimientos}

A S. Viñas Molina y al Departamento de Biblioteca del Hospital Monográfico ASEPEYO de Cirugía Ortopédica, Traumatológica y Rehabilitación de Coslada, Madrid, España.

\section{Bibliografía}

1. Gordon LH, King D. Partial wirst arthrodesis for old un-united fractures of the carpal navicular. Am J Surg. 1961;102:460-4.

2. Inoue G, Tamura Y. Radiolunate and radioscapholunate arthrodesis. Arch Orthop Trauma Surg. 1992;111:333-5.

3. Knirk JL, Jupiter JB. Intra-articular fractures of the distal end of the radius in young adults. J Bone Joint Surg Am. 1986;68:647-59.

4. Strauch RJ. Scapholunate advanced collapse and scaphoid nonunion advanced collapse arthritis. Update on evaluation and treatment. J Hand Surg Am. 2011;36:729-35.

5. Watson HK, Ballet FL. The SLAC wirst: Scapholunate advanced collapse pattern of degenerative arthritis. J Hand Surg Am. 1984;9:358-65.

6. Watson HK. Limited wirst arthordesis. Clin Orthop Relat Res. 1980;149:126-36.

7. Biswas D, Wysocki RW, Cohen MS, Fernandez JJ. Radioscapholunate arthodesis with compression screw and local autograft. J Hand Surg Am. 2013;38:788-94.

8. Hevia-Campomanes E, Miranda F, VivancoC, Gómez F. Los accidentes de trabajo y las enfermedades profesionales. En: Gestión, prestaciones, procedimiento y jurisprudencia. Madrid: Colex; 1990.

9. Berger RA, Bishop AT, Bettinger PC. New dorsal capsulotomy for the surgical exposure of the wrist. Ann Plast Surg. 1995;35:54-9.

10. Murray PM. Radioscapholunate arthrodesis. Hand Clin. 2005;21:561-6.

11. Mühldorfer-Fodor M, Ha HP, Hohendorff B, Prommersberger KJ, van Schoonhoven J. Results after radioscapholunate arthrodesis with or without resection of the distal scaphoid pole. J Hand Surg Am. 2012;37:2233-9.

12. Nalebuff EA, Feldon PG, Millender LH. Rheumatoid arthritis in the hand and wirst. En: Green DP, editor. Operative hand surgery. 2nd ed. New York: Churchill Livingstone; 1988. p. $1655-766$.

13. García-Elías M, Lluch A, Ferreres A, Papini-Zorli I, Rahimtoola Z. Treatment of radiocarpal degenerative osteoarthritis by radioscapholunate arthordesis and distal scaphoidectomy. J Hand Surg Am. 2005;30:8-15.

14. Holleran AM, Quigley RJ, Rafijah GH, Lee TQ. Radioscapholunate arthrodesis with excision of the distal scaphoid: Comparision of contact characteristics to the intact wirst. J Hand Surg Am. 2013;38:706-11.

15. Watson HK, Goodman ML, Johnson TR. Limited wirst arthrodesis, part II: Intercarpal and radiocarpal combinations. J Hand Surg Am. 1981;6:223-33.

16. Bach AW, Almquist EE, Newman DM. Proximal row fusión as a solution for radiocarpal arthritis. J Hand Surg Am. 1991;16:424-31.

17. Sturzenegger M, Buchler U. Radio-scapho-lunate partial wrist arthrodesis following comminuted fractures of the distal radius. Ann Chir Main Memb Super. 1991;10:207-16. 